\title{
New targeted therapies in spondyloarthritis: what are the limits?
}

\author{
Daniel Wendling*, \\ ${ }_{1}^{1}$ Professor of Rheumatology, Head of Department; Department of Rheumatology, University Hospital of Besançon \& EA 4266 \\ EPILAB, University of Franche-Comté, F-25030 Besançon, France \\ *Author for correspondence: dwendling@chu-besancon.fr
}

\begin{abstract}
"G It would be interesting to acquire knowledge about predictive factors of good clinical response for each of these treatments. This would allow a more accurate decision on treatment initiation and a trend to personalized medicine. This may be achieved by retrospectively analyzing the data of randomized controlled trials and their extensions, as well as those from real-life cohorts."
\end{abstract}

First draft submitted: 9 January 2017; Accepted for publication: 27 February 2019; Published online: 28 March 2019

Keywords: anti-IL-17 • anti-IL-23 • anti-TNF • JAK inhibitors • spondyloarthritis • treatment

Spondyloarthritis is a multifaceted chronic inflammatory disease, with predominantly axial involvement (sacroiliac joints and spine), with the possibility of peripheral involvement (synovitis, enthesitis and dactylitis) and extra-articular manifestations (uveitis, inflammatory bowel disease and psoriasis) [1-3]. The terminology of spondyloarthritis covers the cases of axial spondyloarthritis, with radiographic involvement of sacroiliac joints (radiographic axial spondyloarthritis = ankylosing spondylitis), or without radiographic involvement of sacroiliac joints (nonradiographic axial spondyloarthritis) and the cases of peripheral spondyloarthritis. Extra-articular features may be present in each of these subsets, particularly psoriasis; therefore, psoriatic arthritis is part of the spondyloarthritis domain. So, spondyloarthritis may exhibit several phenotypes based on the clinical and radiographical presentation, with global common classification criteria [2]. The therapeutic arsenal of spondyloarthritis has significantly expanded in recent years, both for axial and peripheral forms, largely due to developments in immunopathological knowledge, thus allowing the use of targeted therapies. This has been the case for anti-TNF agents that, for more than 15 years, have shown their interest and transformed the management and outcome of patients with ankylosing spondylitis, as well as peripheral and nonradiographic axial forms of spondyloarthritis. There are currently five anti-TNF agents, with the availability of several biosimilars for three of them. However, it appeared over time that the effect of anti-TNF agents could be different on certain extra-articular manifestations (lack of efficacy of the soluble receptor on inflammatory bowel disease (IBD), lower efficacy on uveitis) $[2,4,5]$. Moreover, these biodrugs may be at the origin of so-called paradoxical effects. These are characterized by the occurrence or exacerbation under anti-TNF therapy of manifestations usually improved by this treatment, even though the rheumatologic disease for which anti-TNF was prescribed is controlled by the latter [6]. This has been described for psoriasis, inflammatory bowel disease and uveitis, particularly.

The arguments in favor of the involvement of the IL-23/Th17 axis in the different forms of spondyloarthritis have paved the way for the development of biomedicines targeting these two cytokines (IL-23 and IL-17) [7]. Thus, there is now approval for IL-17A anti-inflammatory drugs in psoriatic arthritis (secukinumab, ixekizumab; and skin psoriasis) and in ankylosing spondylitis (secukinumab), with developments in other indications (nonradiographic forms of axial spondyloarthritis in particular) and with other IL-17-targeted drugs (bimekizumab, an anti-IL-17A and anti-IL-17F) [8]. The same is true for the targeting of IL-23. This cytokine comprises two subunits: a P40 subunit common with IL-12, and a P19 subunit specific to the IL-23 cytokine. Therefore, IL-23 can be targeted with an anti-P40 antibody (ustekinumab) or anti-P19 antibodies (guselkumab, risankizumab). These approaches have shown their effectiveness in psoriasis and psoriatic arthritis (with approval for some in these indications), and with ongoing assessments in IBD. However, the targeting of IL-23, either with anti-P40 or anti-P19, was unable to show significant improvement in the axial expression of the disease (ankylosing spondylitis) [9,10] and developments

Future $\because$ Medicine 
of these biodrugs have been interrupted in this indication. This inefficiency is in line with the failure of anti-IL-6 receptor biologic agents $[11,12]$ in ankylosing spondylitis, whereas a rationale for this use was available since IL-23 works in synergy with IL- 6 and TGF- $\beta$ for the polarization of native T cells toward Th17 cells producing the IL-17 cytokine family.

A different effect is also observed between anti-IL-17 and anti-IL-23 biologic agents concerning digestive inflammatory disease: whereas a partial efficacy is observed with anti-IL-23, there is a lack of efficacy of anti-IL17 [8], and a placebo-controlled proof-of-concept study was prematurely stopped due to worsening of inflammatory bowel disease in patients treated with anti-IL-17A monoclonal antibody, compared with patients in the placebo group.

This lack of homogeneity of the therapeutic response on the different aspects of the disease, according to the target of inhibition, does not necessarily call into question the conceptual unity of spondyloarthritis and the extra-articular manifestations associated with it, but illustrates the limits of extrapolation from pathophysiological knowledge to therapeutic strategies. Biological arguments are not always confirmed by clinical trials. For IL-23/IL-17 differences of efficacy according to the subset of disease, several hypotheses can be proposed; immunopharmacological considerations, IL-17 production by non-Th17 cells, IL-23-independent production of IL-17 by some cell subsets, time- and tissue-specific cytokine response (tissue distribution of cells expressing IL-23 receptors) may also be taken into account [13]. This raises a reflection on alternatives of inflammation pathways (in particular cellular populations) according to patients' or disease phenotypes. In other words, the several phenotypic presentations of the disease may have different underlying inflammatory and immunological pathways. Such differences may also be present chronologically, with different actors involved at the initiation of the disease at one hand and in the subsequent chronic phase at the other hand.

JAK inhibitors are small molecules, orally administered and represent a new therapeutic class of targeted synthetic disease-modifying anti-rheumatic drugs, with a large number of molecules and potential implications in multiple inflammatory diseases, particularly the spectrum of spondyloarthritis (rheumatologic manifestations, but also psoriasis and inflammatory bowel diseases). Their mode of action is based on the interference with the intracellular JAK-STAT signaling pathway, and in turn, with activity of several proinflammatory cytokines implicated in the disease. Interesting results are available so far in psoriatic arthritis with tofacitinib (a JAK-1 and JAK-3 inhibitor) and filgotinib (a JAK-1 inhibitor). In the same way, early results have been reported in ankylosing spondylitis with these two JAK inhibitors [14-16] and require confirmation. Development is planned in these two indications (psoriatic arthritis and ankylosing spondylitis) with another JAK inhibitor, upadacitinib (ClinicalTrials.gov Identifier: NCT03178487). With this therapeutic class as well, the results seem to be influenced by the phenotypic presentation, more marked on the joint damage than on the skin [17], and on the peripheral compared with the axial involvement, according to the first results of the studies. This will need to be confirmed at a later date.

\section{Conclusion}

It is interesting for the practitioner to multiply the targets and thus broaden the therapeutic range of spondyloarthritis. However, new therapeutic options based on pathogenic hypotheses are not always confirmed by methodologically correct studies, and efficacy is not always the same for all disease presentations.

At the same time, this multiplicity allows for a more personalized therapeutic strategy with differences to be considered in the choice of treatments. This multiplicity of treatment targets underlines the importance of thinking in terms of clinical phenotype of the disease. This may drive current or future updated recommendations about management of spondyloarthritis [18].

\section{Future perspective}

How can the currently available targeted treatment options fit into a comprehensive and 'evidence-based' strategy in a multifaceted disease? To answer this question, we must face some unmet needs.

Whatever the treatment is, there is a need for the availability of reliable, easy to use, biomarkers reflecting or associated to disease activity, with sensitivity to change. Such a biomarker (ideally reflecting pathophysiological mechanisms) would allow to assess with objective methods disease activity (for justification of initiation of a new treatment) at one hand, and therapeutic response (therapeutic tuning in a 'treat to target' strategy) at the other hand. 
It would be interesting to acquire knowledge about predictive factors of good clinical response for each of these treatments. This would allow a more accurate decision on treatment initiation and a trend to personalized medicine. This may be achieved by retrospectively analyzing the data of randomized controlled trials and their extensions, as well as those from real-life cohorts. Some factors have been individualized for anti-TNF treatments (young age of the patients, early disease, elevated C-reactive protein levels at baseline, presence of inflammatory lesions on MRI), but to date not for anti-IL-17 or JAK inhibitors in this disease. Pharmacogenomic evaluation may also be of interest, looking at single nucleotidic polymorphisms of genes of interest and their association with good or bad therapeutic response, or association with adverse events.

Comparative head-to head study is lacking in this situation. The results of such studies may help to suggest a therapeutic hierarchy and guide the clinician. One study comparing anti-IL-17 versus anti-TNF treatment on radiographic progression in ankylosing spondylitis is ongoing [8], but other situations should be explored.

In the same way, studies on therapeutic strategy are needed: is it better to begin with an anti-TNF or an anti-IL17? What could be the positioning of JAK inhibitors in this situation? Is there a place for combination of disease-modifying anti-rheumatic drugs, biologic and/or synthetic? This should be evaluated with the background of different impact of these drugs according to the several stages and phenotypes of the disease.

Finally, what could be the future new therapeutic targets in spondyloarthritis? Pathophysiological knowledge allows us to speculate on cellular actors, such as innate lymphoid cells, mucosal invariant T cells (MAIT), innate natural killer T cells [19], but also on targeting the gut-spondyloarthritis interplay [20], including microbiota and integrins interactions (but recent case reports made aware about possible exacerbation of arthritis or enthesitis with an anti- $\alpha 4 \beta 7$ integrin treatment in inflammatory bowel disease).

At the end, all this emphasizes the utility of a quest for the right treatment for the right patient to improve the global effectiveness of a treatment strategy. As these new and upcoming treatments are expensive, this would have medicoeconomic implications but could bring many potential benefits.

\section{Financial \& competing interests disclosure}

I declare financial/nonfinancial relationships with Abbvie, Pfizer, Roche Chugai, MSD, UCB, Mylan and occasional interventions from AbbVie, BMS, MSD, Pfizer, Roche Chugai, Amgen, Nordic Pharma, UCB, SOBI, Sanofi Aventis, Novartis, Janssen, Celgene, Hospira, Lilly, Sandoz and Grünenthal. The author has no other relevant affiliations or financial involvement with any organization or entity with a financial interest in or financial conflict with the subject matter or materials discussed in the manuscript apart from those disclosed.

No writing assistance was utilized in the production of this manuscript.

\section{Occasional interventions}

AbbVie, BMS, MSD, Pfizer, Roche Chugai, Amgen, Nordic Pharma, UCB, SOBI, Sanofi Aventis, Novartis, Janssen, Celgene, Hospira, Lilly, Sandoz and Grünenthal.

Indirect interests

Abbvie, Pfizer, Roche Chugai, MSD, UCB and Mylan.

\section{References}

1. Wendling D, Claudepierre P, Prati C, Dougados M. Spondyloarthritis: a concept or a disease? Joint Bone Spine 82(6), 387-389 (2015).

2. Sieper J, Poddubnyy D. Axial spondyloarthritis. Lancet 390(10089), 73-84 (2017).

3. Taurog JD, Chhabra A, Colbert RA. Ankylosing spondylitis and axial spondyloarthritis. N. Engl. J. Med. 374(26), 2563-2574 (2016).

4. Maxwell LJ, Zochling J, Boonen A et al. TNF-alpha inhibitors for ankylosing spondylitis. Cochrane Database Syst. Rev. 4, CD005468 (2015).

5. Sepriano A, Regel A, van der Heijde D et al. Efficacy and safety of biological and targeted-synthetic DMARDs: a systematic literature review informing the 2016 update of the ASAS/EULAR recommendations for the management of axial spondyloarthritis. RMD Open 3(1), e000396 (2017).

6. Wendling D, Prati C. Paradoxical effects of anti-TNF- $\alpha$ agents in inflammatory diseases. Expert Rev. Clin. Immunol. 10(1), 159-169 (2014).

7. Wendling D, Guillot X, Prati C. The IL-23/Th17 pathway in spondyloarthritis: the royal road? Joint Bone Spine 82(1), 1-4 (2015).

8. Wendling D, Verhoeven F, Prati C. Anti-IL-17 monoclonal antibodies for the treatment of ankylosing spondylitis. Expert Opin. Biol. Ther. 19(1), 55-64 (2019). 
9. Deodhar A, Gensler LS, Sieper J et al. Three multicenter, randomized, double-blind, placebo-controlled studies evaluating the efficacy and safety of ustekinumab in axial spondyloarthritis. Arthritis Rheumatol. Sep 18. doi: 10.1002/art.40728. [Epub ahead of print] (2018).

10. Baeten D, Østergaard M, Wei JC et al. Risankizumab, an IL-23 inhibitor, for ankylosing spondylitis: results of a randomised, double-blind, placebo-controlled, proof-of-concept, dose-finding Phase 2 study. Ann. Rheum. Dis. 77(9), 1295-1302 (2018).

11. Sieper J, Braun J, Kay J. Sarilumab for the treatment of ankylosing spondylitis: results of a Phase II, randomised, double-blind, placebo-controlled study (ALIGN). Ann. Rheum. Dis. 74(6), 1051-1057 (2015).

12. Sieper J, Porter-Brown B, Thompson L, Harari O, Dougados M. Assessment of short-term symptomatic efficacy of tocilizumab in ankylosing spondylitis: results of randomised, placebo-controlled trials. Ann. Rheum. Dis. 73(1), 95-100 (2014).

13. Siebert S, Millar NL, McInnes IB. Why did IL-23p19 inhibition fail in AS: a tale of tissues, trials or translation? Ann. Rheum. Dis. Oct 8. annrheumdis-2018-213654. doi: 10.1136/annrheumdis-2018-213654. [Epub ahead of print] (2018).

14. Veale DJ, McGonagle D, McInnes IB et al. The rationale for Janus kinase inhibitors for the treatment of spondyloarthritis. Rheumatology (Oxford). Apr 3. doi: 10.1093/rheumatology/key070. [Epub ahead of print] (2018).

15. van der Heijde D, Deodhar A, Wei JC et al. Tofacitinib in patients with ankylosing spondylitis: a Phase II, 16-week, randomised, placebo-controlled, dose-ranging study. Ann. Rheum. Dis. 76(8), 1340-1347 (2017).

16. van der Heijde D, Baraliakos X, Gensler LS et al. Efficacy and safety of filgotinib, a selective Janus kinase 1 inhibitor, in patients with active ankylosing spondylitis (TORTUGA): results from a randomised, placebo-controlled, Phase II trial. Lancet 392(10162), 2378-2387 (2018).

17. Colbert RA, Ward MM. JAK inhibitors taking on psoriatic arthritis. N. Engl. J. Med. 377(16), 1582-1584 (2017).

18. Wendling D, Lukas C, Prati C et al. 2018 update of French Society for Rheumatology (SFR) recommendations about the everyday management of patients with spondyloarthritis. Joint Bone Spine 85(3), 275-284 (2018).

19. Wendling D, Prati C. Spondyloarthritis: an expanding cast of cellular actors. Joint Bone Spine 85(1), 1-3 (2018).

20. Wendling D. The gut in spondyloarthritis. Joint Bone Spine 83(4):401-405 (2016). 